\title{
Analysis of Electromagnetic Wave Interactions on Nonlinear Scatterers using Time Domain Volume Integral Equations
}

\author{
Hüseyin Arda Ülkü ${ }^{(1,2)}$, Sadeed Bin Sayed*(1), and Hakan Bagci ${ }^{(1)}$
}

(1) Division of Computer, Electrical, and Mathematical Sciences and Engineering King Abdullah University of Science and Technology (KAUST), Thuwal, KSA

(2) Department of Electronics Engineering

Gebze Institute of Technology, Kocaeli, Turkey

Effects of material nonlinearities on electromagnetic field interactions become dominant as field amplitudes increase. A typical example is observed in plasmonics, where highly localized fields "activate" Kerr nonlinearities. Naturally, time domain solvers are the method of choice when it comes simulating these nonlinear effects.

Oftentimes, finite difference time domain (FDTD) method is used for this purpose. This is simply due to the fact that explicitness of the FDTD renders the implementation easier and the material nonlinearity can be easily accounted for using an auxiliary differential equation (J.H. Green and A. Taflove, Opt. Express, 14(18), 8305-8310, 2006). On the other hand, explicit marching on-in-time (MOT)-based time domain integral equation (TDIE) solvers have never been used for the same purpose even though they offer several advantages over FDTD (E. Michielssen, et al., ECCOMAS CFD, The Netherlands, Sep. 5-8, 2006). This is because explicit MOT solvers have never been stabilized until not so long ago.

Recently an explicit but stable MOT scheme has been proposed for solving the time domain surface magnetic field integral equation (H.A. Ulku, et al., IEEE Trans. Antennas Propag., 61(8), 4120-4131, 2013) and later it has been extended for the time domain volume electric field integral equation (TDVEFIE) (S. B. Sayed, et al., Pr. Electromagn. Res. S., 378, Stockholm, 2013). This explicit MOT scheme uses predictor-corrector updates together with successive over relaxation during time marching to stabilize the solution even when time step is as large as in the implicit counterpart.

In this work, an explicit MOT-TDVEFIE solver is proposed for analyzing electromagnetic wave interactions on scatterers exhibiting Kerr nonlinearity. Nonlinearity is accounted for using the constitutive relation between the electric field intensity and flux density. Then, this relation and the TDVEFIE are discretized together by expanding the intensity and flux using half and full Schubert-Wilton-Glisson (SWG) functions, respectively. Equations are Galerkin tested in space and the resulting semi-discrete system is integrated in time for the unknown expansion coefficients using the aforementioned predictor-corrector scheme. The explicitness of the MOT scheme allows for incorporation of the nonlinearities as simple discretized function evaluations on the right hand side of the system.

Numerical results that demonstrate the accuracy, efficiency, and applicability of the proposed nonlinear MOT-TDVIE solver will be presented. 